\title{
A Control Method for Hybrid Tilting Systems using Tilting Beams and Air Spring Inclination
}

\author{
Shogo KAMOSHITA \\ Senior Researcher, \\ Kimiaki SASAKI \\ Senior Researcher, \\ Vehicle Noise \& Vibration Laboratory, Vehicle Structure Technology Division \\ Hirohiko KAKINUMA \\ Vice-President, \\ Hokkaido Railway Company
}

\author{
Iwao SATO \\ General Manager, \\ Yorimitsu SATO \\ Research and Development Dept., Railway Operations Headquarters, Hokkaido Railway Company
}

\author{
Satoko NAKAGAKI \\ System Engineering Section, \\ Project Engineering Department, \\ Engineering Division, Rolling Stock Company, Kawasaki Heavy Industries, Ltd.
}

To further increase the speed of railway vehicles running on existing narrow-gauge track, we developed a new tilting system that enables the car body to tilt at a larger angle than conventional tilting trains. The system coordinates tilting beams and a controlled air spring height, thus reducing losses in wheel-load and avoiding lateral displacement of the car body caused by the larger tilting angle. This paper reports the features of the hybrid tilting system, the control method applicable to such tilting and the results of the bench tests carried out.

Keywords: hybrid tilting system, electro-hydraulic actuator, air spring inclination, coordinated tilting control, tilting train

\section{Introduction}

Technologies for the improvement of maximum running speeds, accelerating/decelerating forces and curving performance are conceivable as a major strategy to reduce train travel times. Developments put into practical use so far including a truck with a six-degree inclination that features a newly developed bearing guide ${ }^{1)}$ and a self-steering function using a mechanical link ${ }^{2)}$ developed by JR Hokkaido to sustain passenger ride comfort and decrease lateral forces on curves.

To further increase the speed of railway vehicles running on existing narrow-gauge track, JR Hokkaido developed a new hybrid tilting system that coordinates tilting beams and air-spring inclination. This system is able to reduce losses in wheel-load and avoid lateral displacement of the car body caused by the tilting angle being larger than conventional tilting beam inclination. Moreover, the degree of the reduction of the car body crosssection is less because the overlap with the limit for rolling stock moving dimensions according to the incline operation. However, it is actually difficult for air spring inclination to achieve a larger angle than conventional tilting beam inclination due to the restrictions posed by air spring displacement.

With this in mind, we have developed a new tilting system that can incline up to eight degrees using the sixdegree inclination of the tilting beam that operates with a bearing guide and the two-degree inclination obtained through air spring height control. We have also designed an application for element technologies from the nextgeneration tilt control system ${ }^{3)}$ and a control method for coordination with air spring height control. It is assumed that this hybrid tilting system can secure a level of ride comfort equivalent to that of regular tilting vehicles, even with the improved curving speed.

The Railway Technical Research Institute was responsible for the route position detector, the JT pattern generator and the electro-hydraulic actuator included as components of the system, while Kawasaki Heavy Industries was responsible for the hybrid tilting controller and the air-spring controller, and both parties carried out bench tests together. This paper outlines the results of bench tests involving one truck unit with a mock body, as well as those obtained using a real car body.

\section{Equipment composition of the hybrid tilting system}

\subsection{Inclination through air-spring height control}

JR Hokkaido and Kawasaki Heavy Industries jointly developed a car body inclination control method using airspring height control (currently in practical use on JR Hokkaido's type 201 and 261 diesel railway vehicles ${ }^{4)}$ ), and applied it to the hybrid tilting system reported here. 


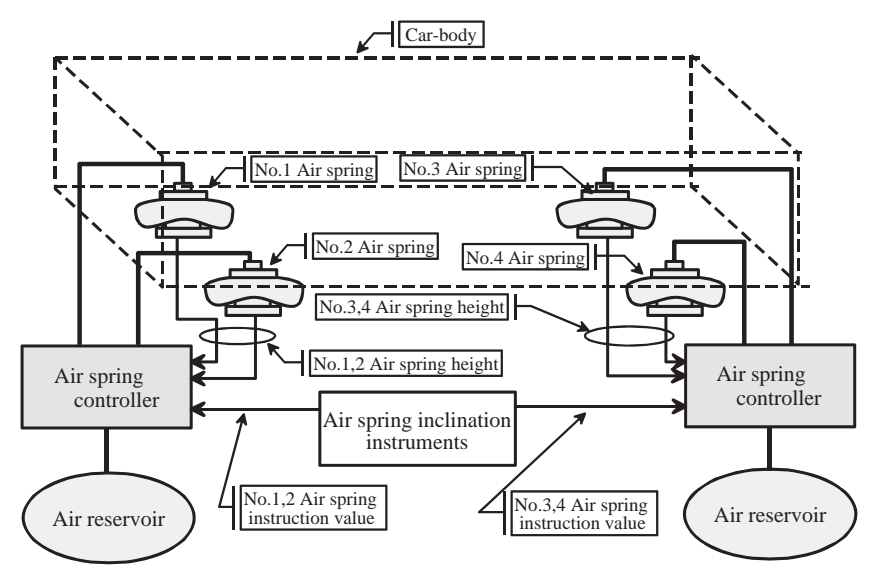

Fig. 1 Air-spring inclination control system

This method involves a system that raises the height of the outer-rail-side air spring when the train runs on curves, and inclines the car body towards the top as a fulcrum of the inner-rail-side air spring. In type 201 and 261 vehicles, however, the condition during curve running is judged without detecting the running position. This is done by processing output from an inertia sensor on the car body, and the vehicles then operate car body inclination from the running condition and the shape of the railway track.

Control of the air spring height compares the instruction value from the upper controller with the real displacement of the air spring, and the valves are operated according to the deviation and supply or exhaust air from inside the air spring. Figure 1 outlines the control system. The height of the air spring is measured using the vehicle height sensor installed in the leveling valve. The air-spring controller supplies or exhausts compressed air from inside the air spring according to the amount of deviation of the instruction value and the obtained air spring height. The air springs of the outer rail increase the height on curve sections. The usual leveling valve function is discontinued, and the height of the air spring is controlled electrically during car body inclination. If problems occur for any reason, the system shifts to mechanical levelcontrol mode, and can compensate for the problem function as a conventional leveling valve. Inclination of up to two degrees is made by this air spring operation.

\subsection{Tilting-beam inclination using an EHA}

The next-generation tilt control system uses an electro-hydraulic actuator (referred to below as an $E H A$ ) for the incline operation of the tilting beam. The EHA has an integrated structure combining the hydraulic cylinder with the oil pressure source and the valve unit etc. as shown in Fig. 2. The oil pressure pump can expel and intake from both directions.

The motor drives a pump directly controlling expansion and contraction of oil in the cylinder by means of its rotation's direction and speed. The external connection is therefore composed only of electric wiring, and the hydraulic piping is enclosed in the main body. This structure helps avoiding oil leakage during operation and makes it difficult for contamination to mix in. The actua-

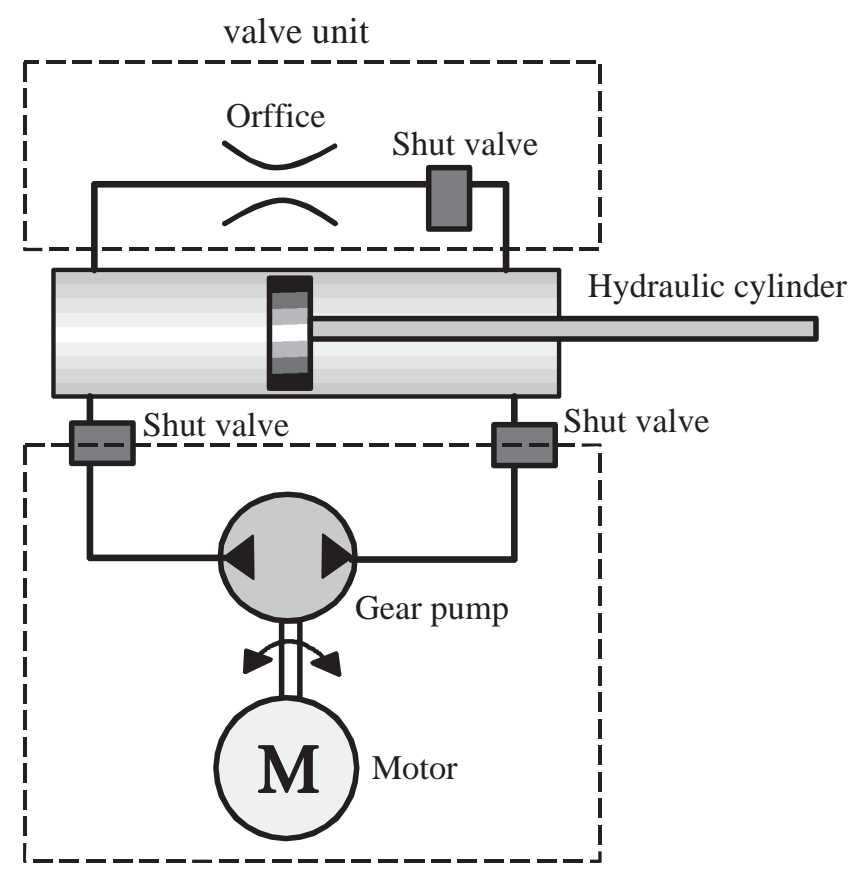

oilpressure source unit

Fig. 2 Hydraulic circuit composition of EHA

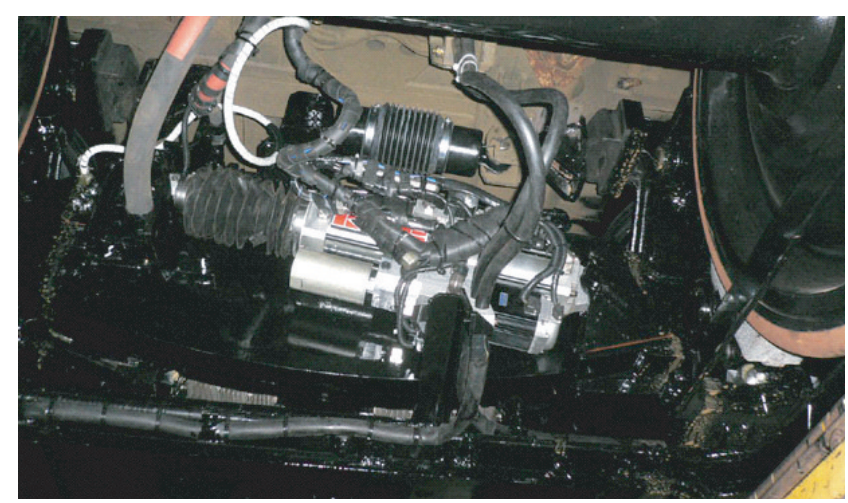

Fig. 3 External view of EHA installed in truck

tor positioning accuracy and response are much better than the conventional pneumatic cylinders used for the existing tilting-beam inclination system. The actuator is fail-safe because it acts as a damper, and performs the same way as a conventional tilting damper by switching the shut-off valve if abnormalities occur during running. As outlined above, the EHA can achieve high response and accurate tilting-beam inclination, offering a fully balanced high level of performance including areas such as maintenance and reliability. Car body tilting operation of six degrees is achieved using the EHA with these features. Figure 3 shows the outside of the EHA installed on the experimental truck.

\subsection{Equipment composition of the hybrid tilting system}

The hybrid tilting system is an integration of the two car body inclination technologies described above. The equipment composition of the overall hybrid tilting system that enables up to eight degrees of inclination is shown in Fig. 4. 


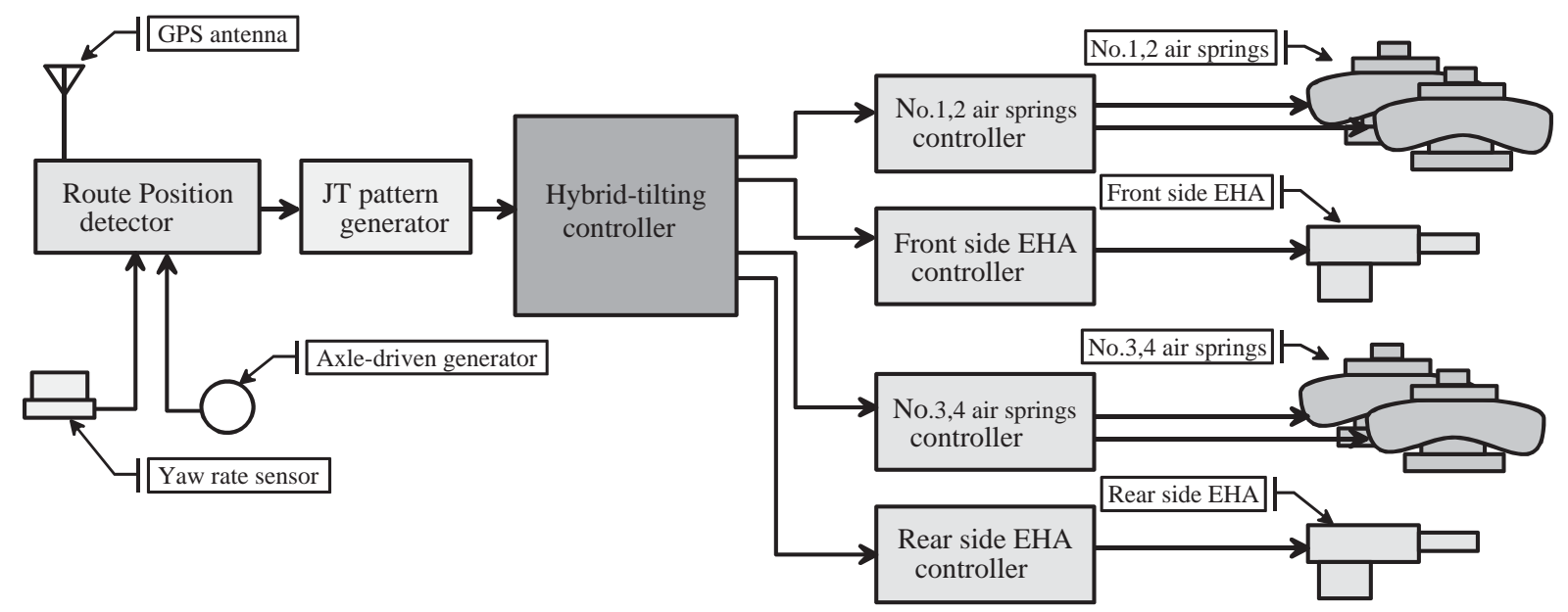

Fig. 4 Equipment composition of hybrid tilting system

The GPS and curvature collation techniques of the next-generation tilting control system ${ }^{5) 6}$ are adapted to the hybrid tilting system for the vehicle's positional detection system. This method detects the train's current position precisely and steadily using a combination of GPS, curvature collation from the onboard database, and the cumulative running distance obtained from an axledriven generator. The system also makes proper use of the best method for detecting the vehicle's current position according to the reliability of the GPS signal. This method is proven to achieve a detection accuracy of approximately $\pm 4[\mathrm{~m}]$, including in tunnel sections where GPS signals are not received.

We also apply a tilting pattern used in the next-generation tilting train as an instruction pattern for the hybrid tilting system. The pattern is calculated to optimize the ride comfort evaluation index on transition curves for tilting trains using values from a curvature and cant database as well as the vehicle's velocity. The evaluation index is called TCT (Transition Curve Total) ${ }^{7}$, and the tilting pattern is known as the JT pattern (a judgment function with TCT). The JT pattern generator creates the instruction tilting angle using data on the running position, vehicle velocity, curvature and cant transmitted from a route-position detector and the pattern is sent to the hybrid tilting controller.

The hybrid tilting controller distributes the inputted total tilting angle to the tilting beam carbody inclination and the air spring inclination, and outputs the instruction pattern to each device. It also receives signals from a train information device and instructs the EHA controllers and air-spring controllers to manage solenoids or shut valves. These devices perform displacement feedback based on the instruction values received from the hybrid tilting controller. All controlling devices monitor each other, and if abnormalities occur the tilting control is immediately stopped. In this case, the EHA is also switched to the state of the tilting damper, and the air springs are changed to a state of mechanical leveling valve operation to promptly secure the natural tilting performance of the vehicle.

\section{Control method for the hybrid tilting system}

\section{$3.1 n$-second advanced JT pattern}

The tilting beam inclination using EHA and the body inclination with the air spring exhibit differences in areas such as the characteristic response, positioning accuracy and the maximum angle of gradient. In particular, it is assumed that the response of air spring could be a problem, because it is akin to controlling a large-diameter pneumatic cylinder with a relatively small valve. From the step response results of air spring operation carried out in the bench test, it was also conceivable that

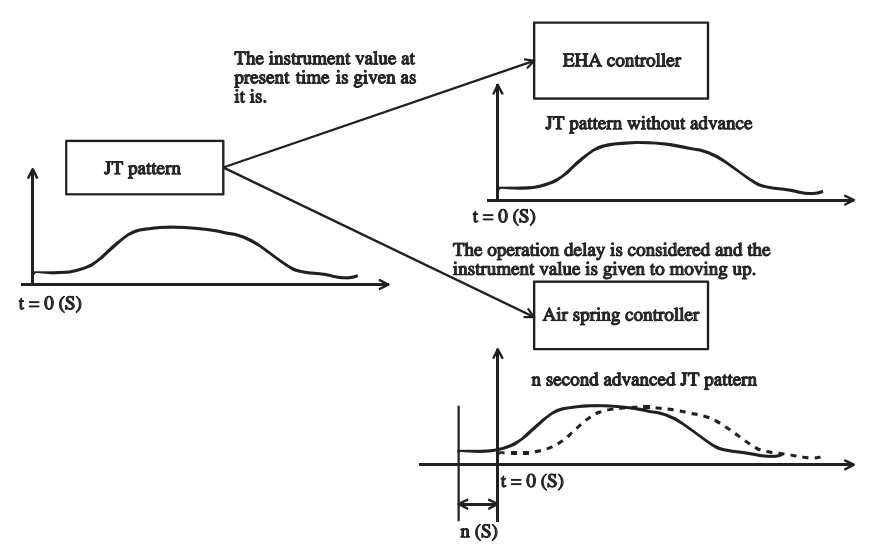

Fig. 5 Examples of JT pattern and advanced JT pattern

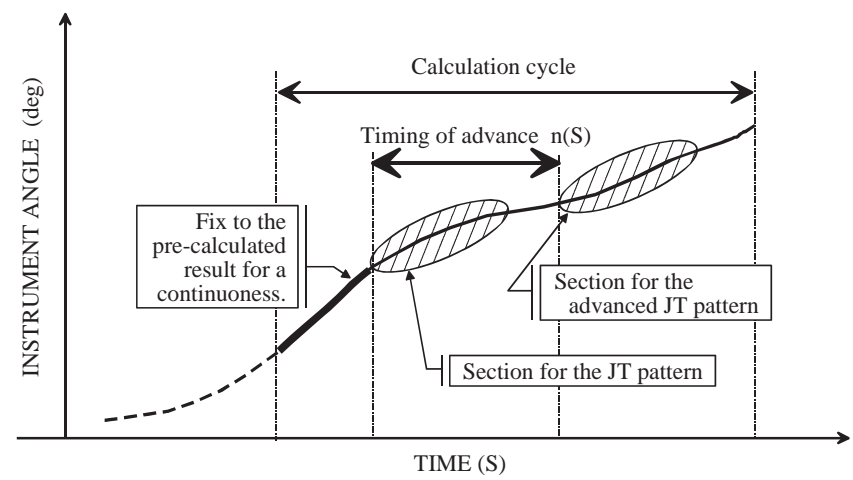

Fig. 6 Relationship between JT pattern and advanced JT pattern 
delayed operation of air spring inclination may prevent JT pattern achievement in hybrid tilting operation. Considering such response of air springs; the JT pattern generator calculates an $n$-second advanced pattern at the same time as calculating the non-advance JT pattern, and uses this to instruct the hybrid tilting controller. Thus, air springs are controlled using an $n$-second advanced JT pattern (Fig. 5).

The JT pattern is calculated continuously in the pattern generator at fixed intervals, and the $n$-second advanced pattern is shifted forward $n$ seconds. Figure 6 shows the relationship between an $n$-second advanced JT pattern and a normal JT pattern. It assumes that the appropriate advanced timing for the air spring inclination will be set up by the bench test described latter.

The air spring inclination operates as a conventional leveling valve device on straight-line sections, and the control mode of the inclination is changed if necessary on curve sections. Neither straight-line nor curve sections are clearly specified in the ground data, which includes curvature and cant at intervals of one meter. The system therefore judges curve sections when the sum of the absolute value of the JT pattern and $n$-second advanced JT pattern surpass the threshold which is set including a hysteresis.

\subsection{Coordination improvement of the car body tilting motion to the JT pattern}

The tilting angle, controlled by the EHA and the air spring, is required to correspond to the JT pattern. The response of the air spring varies according to the quantity of air flow that is supplied and exhausted from air spring, and the control performance of the EHA can be adjusted through factors such as controller gain and the current limit of the servo amplifier. However, it is difficult to fix only one parameter of these controls for uniform inclination response as the robustness depends on the car body's weight variation. In addition, the optimum value for the JT pattern advance outlined in the preceding paragraph, changes according to the condition of the car body. In light of this, in order to obtain an accurate total car body tilting based on a JT pattern, we have studied a control method to compensate a deviation between a JT pattern and total incline angle of the air spring and tilting beam. The deviation is fed back only to the EHA controller as it is superior in response. Figure 7 shows a controller block diagram of the hybrid tilting system. We

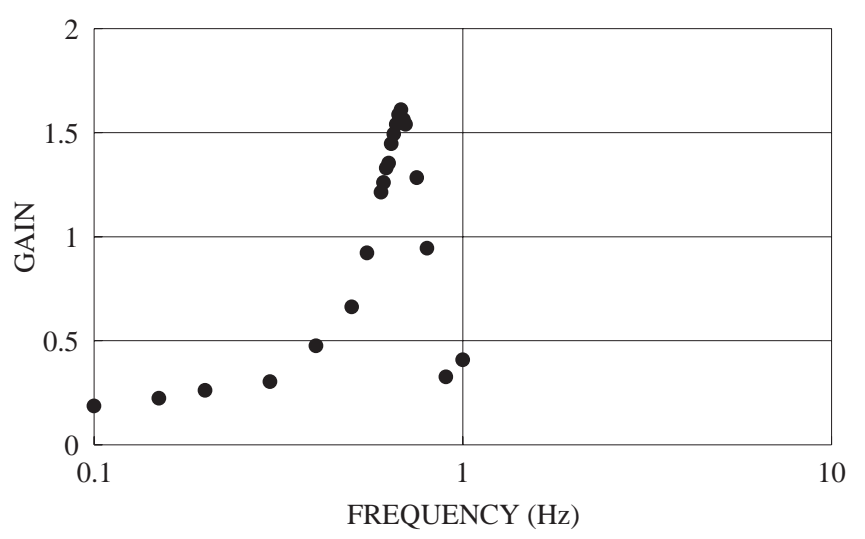

Fig. 8 Frequency response of roll excitation

consider that these controller components enable hybrid tilting control that takes into account variables in the air spring height or dead zones.

\subsection{Controller component for stable control}

In the earlier stage of the bench test on a mock body (outlined in the next section), unstable rolling oscillation initially occurs in the car body. We confirmed that the EHA roll-compensating motion and the air spring's roll-direction vibration interfere with each other. When the air spring moves vertically rolling from side to side in inverse phase with a disturbance, the EHA movement compensates for the rolling deviation. These motions also make up the natural vibration mode of the car body and the air spring. The air spring's natural vibration resonance frequency of rolling is found to be notable at about $0.7[\mathrm{~Hz}]$ according to the results of bench test (Fig. 8). We therefore designed an FIR-type low-pass filter which blocks over-resonance frequency, and attached it to the feedback signal of the air spring's inclination angle. The EHA instruction value for compensation is therefore cut off in high-frequency situations. We have confirmed that the lowpass filter (shown within the dotted line in Fig. 7) settles rolling excitation and stabilizes car body inclination.

\section{Bench test results}

\subsection{Equipment composition of the bench tests}

Figure 9 shows the equipment composition of the bench

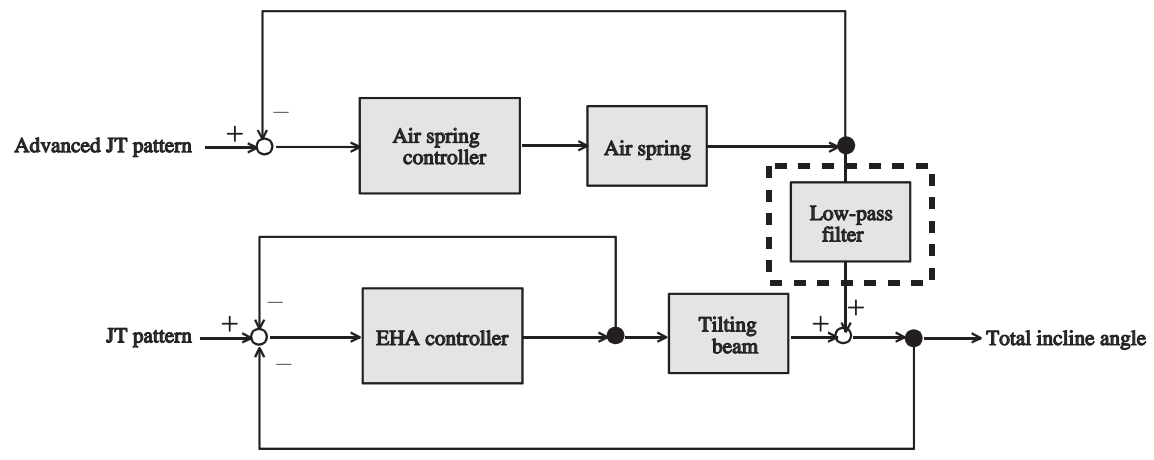

Fig. 7 Controller block diagram 


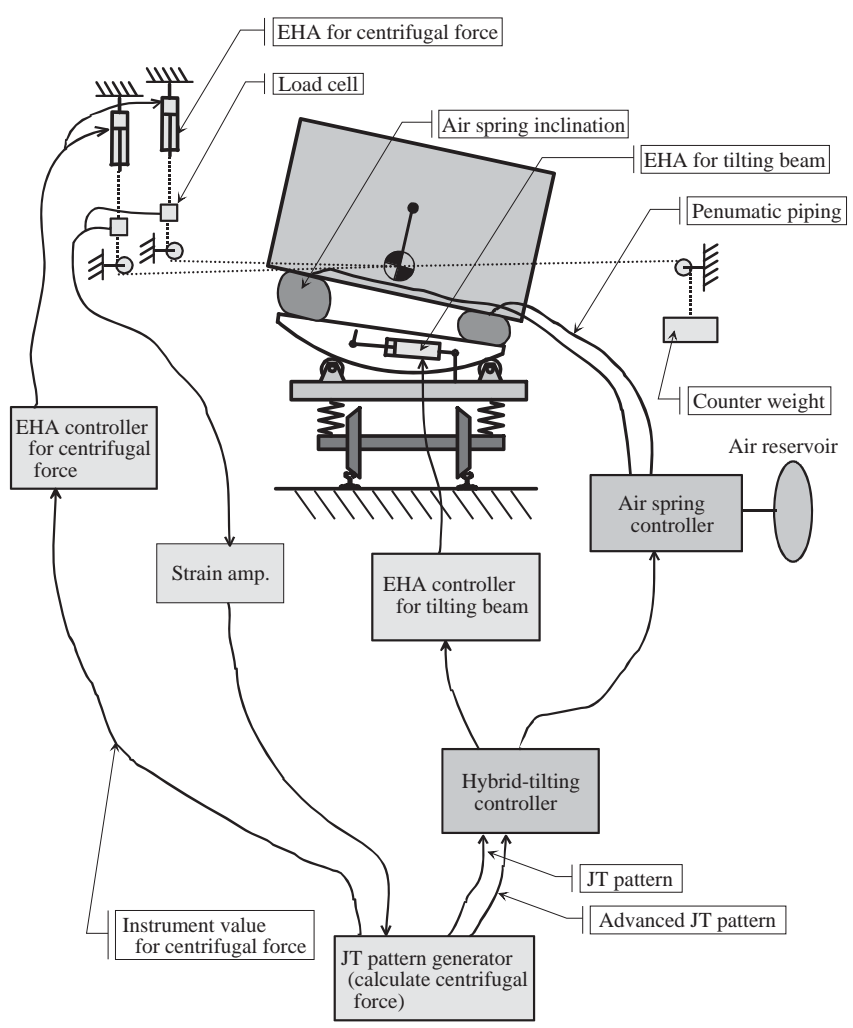

Fig. 9 Composition of equipment for the bench tests

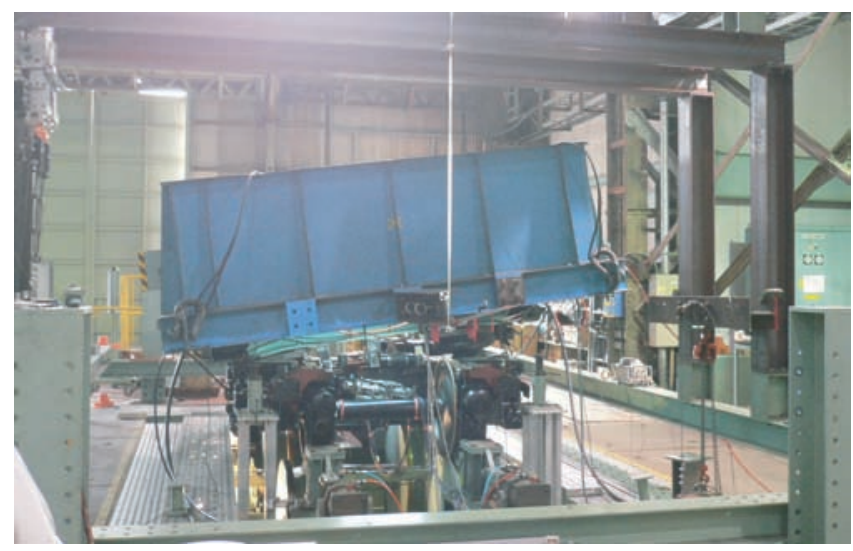

Fig. 10 External view of mock-body bench test

Table 1 Specifications of bench test devices and test vehicle

\begin{tabular}{c|c|c|c}
\hline & Bench test & Type 283 & $\begin{array}{c}\text { Next- } \\
\text { generation } \\
\text { vehicle }\end{array}$ \\
\hline Half car-body weight [kg] & 12608 & 16928 & 13417 \\
\hline $\begin{array}{c}\text { Upper tilting beam weight } \\
{[\mathrm{kg}]}\end{array}$ & 13235 & - & - \\
\hline $\begin{array}{c}\text { Distance from tilting roll } \\
\text { center to center of } \\
\text { gravity [mm] }\end{array}$ & 521 & 555 & 723 \\
\hline
\end{tabular}

tests, and Fig. 10 shows an external view. Table 1 shows the specifications of the bench test devices and the vehicle for use in running tests. The mock body is pulled laterally by another two EHA to simulate the centrifugal force on
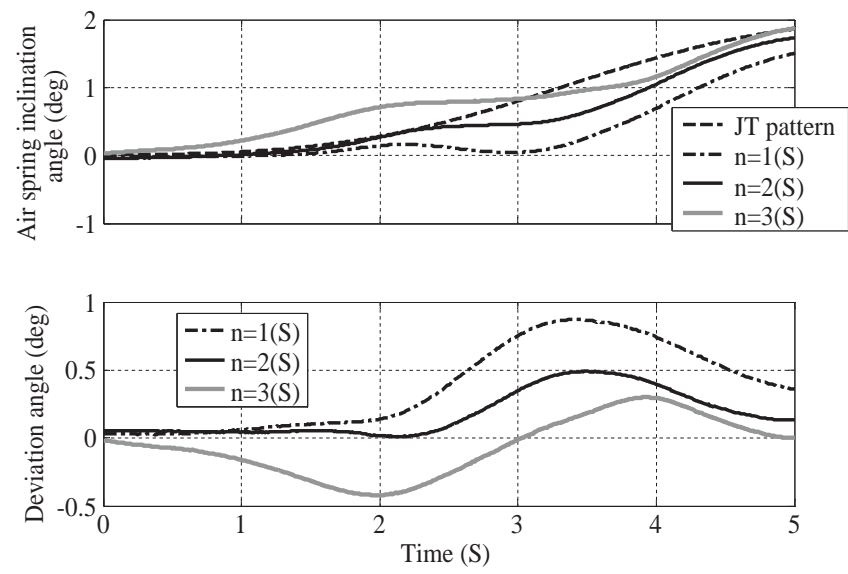

Fig. 11 Air spring inclination response according to advance timing

curve sections. The hybrid tilting control is carried out based on the JT pattern which is calculated from the real railway truck alignments where a test run will be taken place. The JT pattern is generated estimating a running speed of a certain speed up for a test run in the near future. At the same time, the JT pattern generator calculates the lateral centrifugal force acting on the car body using the estimated speed, and controls the lateral force EHA to match the calculated force and the real generated force measured by the load cell. We examined the behavior of the mock body under the experimental conditions as above.

\subsection{Results of hybrid tilting system tests on the mock body}

Figure 11 shows the behavior of car body inclination and the deviation from the instruction angle for different advance timing input to the hybrid tilting controller when a vehicle runs into a transition curve from a straight track. In the case of a three-second advanced pattern, air spring motion begins too early, and the tilting beam controlled by the EHA moves in the opposite direction to the pattern in order to prevent excess car-body tilting to the inner-rail side. In the case of a one-second advanced pattern, however, the air spring's inclination angle is insufficient at the entrance of the curve section. The smallest root mean square of the air spring's inclination angle deviation from the JT pattern at the curve transition is a two-second advanced pattern. Therefore, it is thought that the advance timing of 1.5 to 2.5 seconds is optimal for the specification of this bench test's devices.

Inclination operation was carried out with simulated centrifugal force acting on the car body. Figure 12 shows the situation of the hybrid tilting operation. The total angle of inclination (i.e. the sum of the tilting beam angle and the air spring inclination) closely followed the JT pattern, and it is found that the aimed inclination pattern is successfully achieved.

\subsection{Results of bench tests on the real car body}

Bench tests were carried out using a real car body, with the complete system installed in the truck produced 


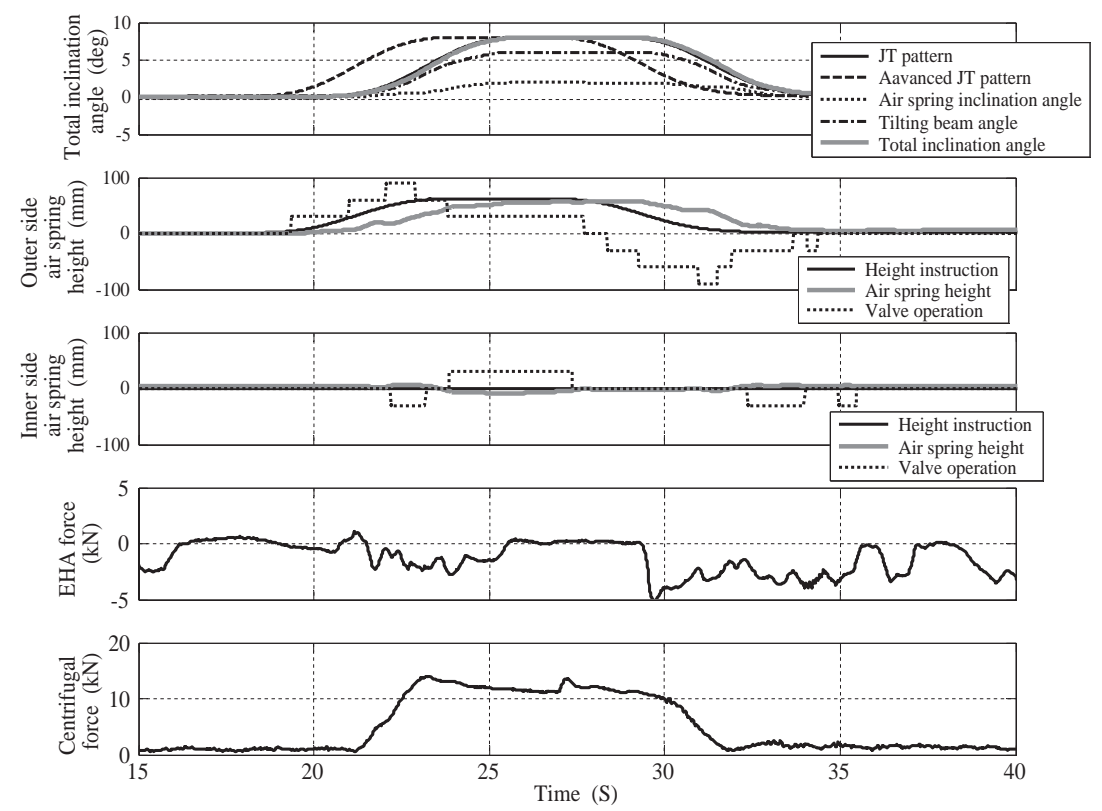

Fig. 12 Incline motion with centrifugal force of mock body

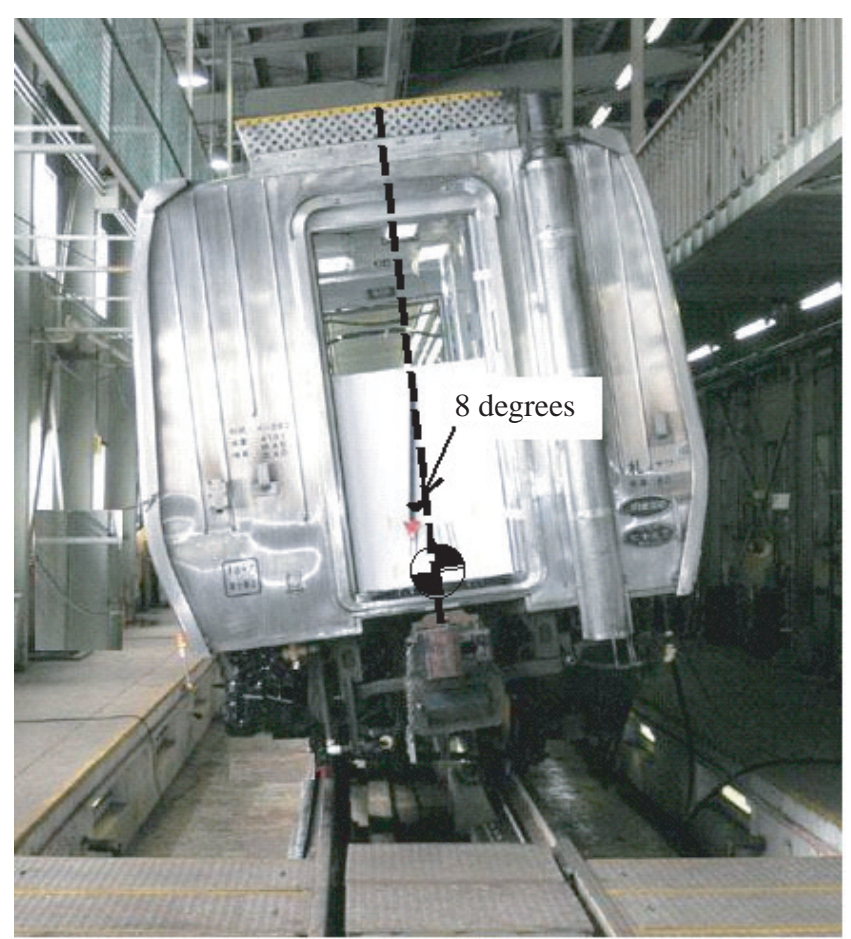

Fig. 13 External view of real car-body bench test

for the hybrid tilting running test. In these real-body bench tests, equipment including a GPS route-position detector and real-time JT pattern generators were activated to confirm the inclination operation. The GPS reception, the axle-driven generator's signal and an inertia sensor data were obtained from a test run executed beforehand, and these data were entered into the route-position detector. Figure 13 shows an external view of the bench test, and Fig. 14 shows the motion of the hybrid tilting inclination. The air spring inclination may cause a steady deflection within the range of its dead zone, and
Fig. 14 shows the compensating operation of the EHA corresponding to the JT pattern (the circles on the right of Fig. 14). Moreover, the EHA does not compensate for high-frequency vibration, which causes system oscillation for the effects of the low-pass filter in the hybrid tilting controller (the circle on the left of Fig. 14). We conducted a series of hybrid tilting control tests without problems, and confirmed hybrid tilting operation corresponding to the running conditions assumed.

\section{Conclusions}

1) We have made a number of findings on the basic operation characteristics of air-spring car-body inclination and tilting-beam inclination using EHA, and have established a method for the coordinated operation of these devices.

2) The advance timing of the JT pattern applied to the air-spring controller was experimentally settled on, and its effects were confirmed.

3) It was confirmed that stable incline operation can be achieved by applying an appropriate low-pass filter to feedback from the gradient angle of the air spring inclination in hybrid tilting operation.

4) Stable hybrid tilting operation was verified through bench tests using a mock body with simulated centrifugal force as well as bench tests on a real truck and car body prepared for running tests.

5) We plan to carry out further running tests on the hybrid tilting system and verify its effects in terms of the ride comfort improvement and the integrity of the overall system.

\section{References}

1) Transport dept. Railway Operation Headquarters 

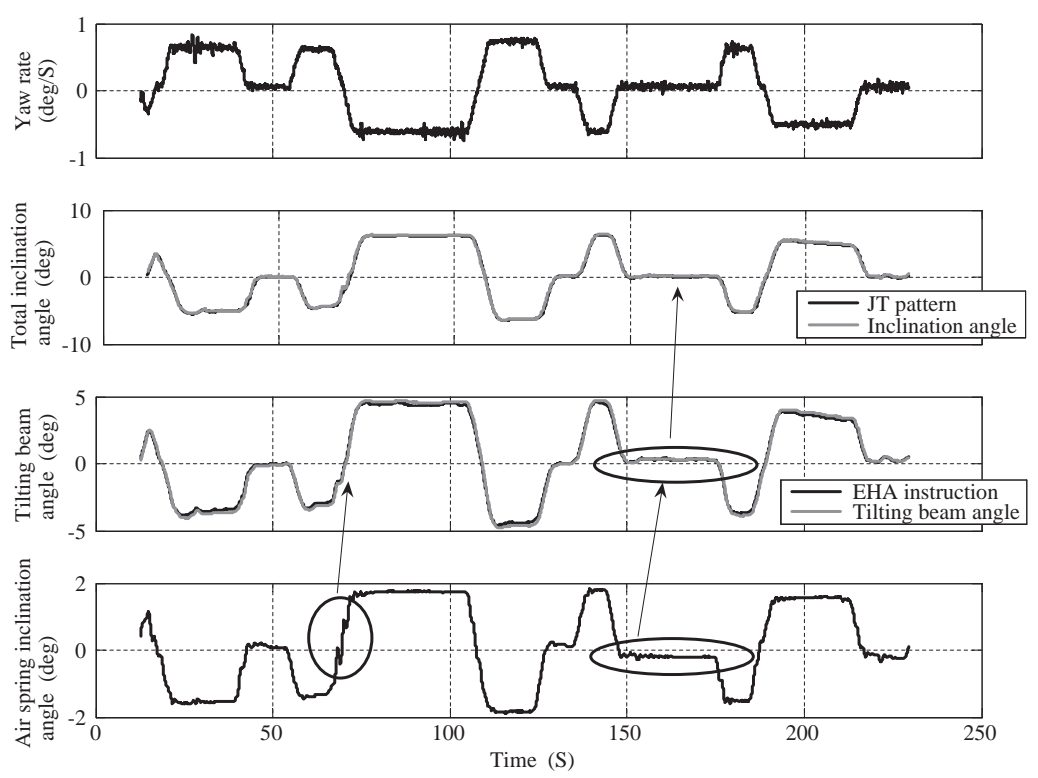

Fig. 14 Hybrid tilting motion of real car-body operation

Hokkaido Railway Company, "JR Hokkaido Type 283 limited express tilting diesel car," Vehicle Technology, Vol.209, 1996 (in Japanese).

2) Sato, E. Kobayashi, H. et al., "Lateral Force between Wheel and Rail during Curve Negotiation of a Limited Express Diesel Car with Forced Steering Bogie," Transaction of the Japan society of mechanical engineers, Vol.64, No.625, pp.315-322, 1998 (in Japanese).

3) Enomoto, M. Kamoshita, S. et al., "Development of Tilt Control System Using Electro-Hydraulic Actuators," QR of RTRI, Vol.46, No.4, pp.219-224, 2005.

4) Hirayama, M. Nakagaki, S. et al., "Running Curve Comfortably at High Speed - Tilting Control System by Use of Air Spring for Rolling Stock - ," KAWASAKI TECHNICAL REVIEW, Vol.160, pp.34-37, 2006 (in Japanese).

5) Sasaki, K., "Position Detection System Using GPS for Carbody Tilt Control," presented at the TRB2005 Annual Meeting, 2005.

6) Umehara, Y. Sasaki, K., "Position Detection System Using GPS for Carbody Tilt Control," presented at the S-TECHO6 Meeting, 2006.

7) Suzuki, H. Shiroto, H. et al., "Psychophysical Evaluation of Railway Vibration Discomfort on Curved Sections," QR of RTRI, Vol.41, No.3, pp.106-111, 2000. 